APPROXIMATE ANALYSIS FOR INTERLAMINAR STRESSES IN COMPOSITE STRUCTURES WITH THICKNESS DISCONTINUITIES

Cheryl A. Rose and James H. Starnes, Jr.

NASA Langley Research Center

Hampton, VA 23681-0001

Presented at the AIAA/ASME/ASCE/AHS/ASC 37th Structures,

Structural Dynamics, and Materials Conference

AIAA Paper No. 96-1497

Salt Lake City, Utah

April 15-17, 1996 
. 


\title{
APPROXIMATE ANALYSIS FOR INTERLAMINAR STRESSES IN COMPOSITE STRUCTURES WITH THICKNESS DISCONTINUITIES
}

\author{
Cheryl A. Rose ${ }^{*}$ and James H. Starnes, Jr. ${ }^{\dagger}$ \\ NASA Langley Research Center \\ Hampton, Virginia 23681-0001
}

\begin{abstract}
$\underline{\text { Abstract }}$
An efficient, approximate analysis for calculating complete three-dimensional stress fields near regions of geometric discontinuities in laminated composite structures is presented. An approximate three-dimensional local analysis is used to determine the detailed local response due to far-field stresses obtained from a global two-dimensional analysis. The stress results from the global analysis are used as traction boundary conditions for the local analysis. A generalized plane deformation assumption is made in the local analysis to reduce the solution domain to two dimensions. This assumption allows out-of-plane deformation to occur. The local analysis is based on the principle of minimum complementary energy and uses statically admissible stress functions that have an assumed through-the-thickness distribution. Examples are presented to illustrate the accuracy and computational efficiency of the local analysis. Comparisons of the results of the present local analysis with the corresponding results obtained from a finite element analysis and from an elasticity solution are presented. These results indicate that the present local analysis predicts the stress field accurately. Computer execution-times are also presented. The demonstrated accuracy and computational efficiency of the analysis make it well suited for parametric and design studies.
\end{abstract}

\section{Introduction}

Advanced composite materials are being used more extensively for aircraft primary structural components. To exploit fully the benefits of composite materials for use in these comonents, a thorough understanding of the corresponding response and failure characteristics of these structures is required. Practical forms of these primary structural components have many local detail features that cause gradients in the stresses and displacements near these detail features. In addition, some structural features have thickness discontinuities that cause local three-dimensional interlaminar stress gradients. Thickness discontinuities commonly occur at

\footnotetext{
"Aerospace Engineer, Structural Mechanics Branch. Member AIAA.

${ }^{\dagger}$ Head, Structural Mechanics Branch. Fellow, AIAA.

Copyright $\odot 1996$ by the American Institute of Aeronautics and Astronautics, Inc. No copyright is asserted in the United States under Title 17. U.S. Code. The U.S. Government has a royalty-free license to exercise all rights under the copyright claimed herein for Governmental Purposes. All other rights are reserved by the copyright owner.
}

flange terminations in a stiffened panel, at dropped plies or skin thickness changes, at the edges of fail-safe straps, and at the edges of lap joints. Local interlaminar stress gradients have been identified as being significant contributors to the failure of composite structures for all loading conditions. An example of such a failure occurs in stiffened panels that are loaded into the postbuckling load range. ${ }^{1}$ Failure can occur for these panels when the high local stress gradients in the interface between the skin and the stiffener flange have stresses that exceed the low interlaminar strength of the composite materials. These stress gradients can cause the stiffeners to separate from the skin.

With few exceptions, ${ }^{2-6}$ previous analyses of laminated plate structures with thickness discontinuities have been conducted using displacement-based finite element analyses. The large number of degrees of freedom required to obtain accurate transverse stress predictions with this analysis method makes the use of this method computationally expensive or even infeasible for structures of practical size and with practical structural design details. This observation is particularly true if the analysis is included in a design or structural optimization code where analyses are performed repetitively or, if nonlinear deformations associated with postbuckling or internal pressure are considered.

The present paper presents an alternative approximate analysis method for determining the three-dimensional interlaminar stresses at a local thickness discontinuity in a laminated composite structure. The analysis is limited to linear elastic material behavior and geometrically linear deformations. The approximate analysis method is based on stress functions and the principle of minimum complementary energy. The method is similar to an approximate analysis method presented by Kassapoglou and DiNicola, ${ }^{5,6}$ but uses the more refined stress function assumptions for each layer of a laminate that were used by Yin ${ }^{7}$ for analyzing the classic free edge problem. In addition, the present analysis includes both the thick and the thin regions of a panel with a thickness discontinuity as shown in Fig. 1. Only the thick region of the panel was modeled in the previous approximate analyses. ${ }^{5,6}$ Including both the thick and thin panel regions and the refined stress assumptions for each layer provides a more accurate stress field prediction, and a better representation of the load transfer between the two regions. Furthermore, the present analysis can model general lam- 
inates and is not restricted to orthotropic layers as are currently available elasticity solutions. ${ }^{2,3,4}$

The flange termination region at a skin-stiffener interface is emphasized in the present paper; however, the method can be applied or extended to other thickness discontinuity configurations that occur in practical composite structures. The computational efficiency of the analysis is demonstrated by solution execution-time results, and the accuracy of the analysis is demonstrated by comparisons of results from the present analysis with results from finite element and elasticity analyses. The results presented herein suggest that the present approximate analysis will provide structural designers with a computationally efficient means for assessing the influence of structural, geometric and material parameters on local three-dimensional stress fields and for designing laminated composite structures with critical local detail features.

\section{Problem Definition and Solution Approach}

The physical problem considered has a single discontinuity, or change in thickness, as shown in Fig. 1. A global-local analysis approach is used for the present analysis to make the analysis general and applicable to a variety of structural configurations. The global analysis consists of a two-dimensional finite element analysis of a structure subjected to prescribed loading and displacement boundary conditions. Stress resultants obtained from the global analysis at a point (or points) of interest in the structure are applied as far-field traction boundary conditions to a local analysis of the thickness discontinuity region. The remainder of the present paper focuses on the details of the local analysis.

It is assumed that the deformations in the local analysis can be represented accurately by the assumption of generalized plane deformations. For this class of problems, the loading, geometric and material properties, and stresses and strains are independent of the longitudinal coordinate $x$ (see Fig. 1 for the definition of the coordinate system). The solution domain can therefore be reduced to any $y-z$ cross-sectional plane that is remote from the ends of the structure where the stresses and strains are influenced by the boundary conditions, and a state of generalized plane deformation may not exist.

Lekhnitskii ${ }^{8}$ investigated the class of deformations for which the stress tensor is independent of the longitudinal coordinate $x$, and found that the most general form of the displacement field for this class of problems (see page 104 of Ref. 8 ) is

$$
\begin{gathered}
u(x, y, z)=x(A y+B z+C)+U(y, z)+u^{\prime} \\
v(x, y, z)=(-I / 2) A x^{2}-\vartheta x z+V(y, z)+v^{\prime} \\
w(x, y, z)=(-1 / 2) B x^{2}+\vartheta x y+W(y, z)+w^{\prime}
\end{gathered}
$$

where $\mathrm{A}$ and $\mathrm{B}$ are the negative bending curvatures in the $x-y$ and $x-z$ planes, respectively, $\mathrm{C}$ is the strain $\varepsilon_{x}$ at $y=0$ and $z=0$ and $\vartheta x$ is the rotation about the $x$ axis. The values of $A, B, C$ and $\vartheta$ are the same for all layers. The functions $U, V$ and $W$ are unknown components of the displacement, and $u^{\prime} v^{\prime}$ and $w^{\prime}$ are rigid body displacements.

A model of a cross-section of the structural detail and the applied loads used in the local analysis are shown in Fig. 2. The stress resultants $N_{y}$ and $M_{y}$ shown in Fig. 2 and the stress resultants $N_{x}, M_{x}$ and $M_{x y}$ are determined from the global analysis. The local solution domain is divided into two regions, $\mathrm{A}$ and $\mathrm{B}$, as shown in Fig. $2 \mathrm{a}$, to account for the change in geometry at the thickness discontinuity. Each region is assumed to be sufficiently long in the $y$-direction so that the solution provided by the global two-dimensional analysis is valid away from the discontinuity. Independent solutions are developed for the two regions, and inter-region continuity conditions obtained from the variational statement are used to connect the two solutions. Region $\mathrm{A}$ includes both the terminated laminate (shown in Fig. 1) and the laminate that continues into region $\mathbf{B}$. Region $\mathbf{A}$ consists of $N$ layers separated by $n$ planar interfaces. An adhesive or bond layer may also be included between the continuous and terminated laminates. Region $\mathrm{B}$ includes only the laminate continued from region $\mathrm{A}$, and consists of $N_{s}$ layers which are separated by $n_{s}$ interfaces. The thickness of the layers are represented by $h^{(k)}=z^{(k-1)}-z^{(k)}$ and are numbered consecutively from the top surfaces of the laminates (see Fig. 2b). Mathematical layers may represent individual plies that are reinforced by a system of parallel fibers oriented at an angle $\theta$ with respect to the $x$ axis or they may represent a portion of the thickness of a ply. The layers are continuous between regions. Each layer (indicated by the subscript $k$ ) is regarded as a homogeneous, monoclinic, linear elastic material, defined by the following constitutive relation

$$
\left\{\begin{array}{c}
\varepsilon_{1} \\
\varepsilon_{2} \\
\varepsilon_{3} \\
\varepsilon_{4} \\
\varepsilon_{5} \\
\varepsilon_{6}
\end{array}\right\}=\left[\begin{array}{cccccc}
S_{11} & S_{12} & S_{13} & 0 & 0 & S_{16} \\
S_{12} & S_{22} & S_{23} & 0 & 0 & S_{26} \\
S_{13} & S_{23} & S_{33} & 0 & 0 & S_{36} \\
0 & 0 & 0 & S_{44} & S_{45} & 0 \\
0 & 0 & 0 & S_{45} & S_{55} & 0 \\
S_{16} & S_{26} & S_{36} & 0 & 0 & S_{66}
\end{array}\right]^{(k)}\left\{\begin{array}{l}
\sigma_{1} \\
\sigma_{2} \\
\sigma_{3} \\
\sigma_{4} \\
\sigma_{5} \\
\sigma_{6}
\end{array}\right\}^{(k)}
$$

where contracted notation has been used; e.g.,

$$
\left[\sigma_{x x}, \sigma_{y y}, \sigma_{z z}, \sigma_{y z}, \sigma_{x z}, \sigma_{x y}\right]^{T}=\left[\sigma_{1}, \sigma_{2}, \sigma_{3}, \sigma_{4}, \sigma_{5}, \sigma_{6}\right]^{T}
$$

The corresponding notation is used for the strains. For generalized plane deformation, a longitudinal strain $\varepsilon_{l}^{(k)}$ can be specified. Expressing the longitudinal stress $\sigma_{1}^{(k)}$ in terms of this strain and the remaining 
stress components gives

$\sigma_{1}^{(k)}=\left(\varepsilon_{1}^{(k)}-S_{1 j}^{(k)} \sigma_{j}^{(k)}\right) / S_{11}^{(k)} \quad(j=2,3,6)$

In Eq. (4) and the equations that follow, Cartesian tensor summation rules are assumed unless otherwise noted. Substituting Eq. (4) into the right-hand side of Eq. (2) and replacing the first row by the above expression for $\sigma_{1}^{(k)}$ gives

$$
\left\{\begin{array}{l}
\sigma_{1} \\
\varepsilon_{2} \\
\varepsilon_{3} \\
\varepsilon_{4} \\
\varepsilon_{5} \\
\varepsilon_{6}
\end{array}\right\}^{(k)}\left[\begin{array}{cccccc}
\frac{1}{S_{11}} & \frac{-S_{12}}{S_{11}} & \frac{-S_{13}}{S_{11}} & 0 & 0 & \frac{-S_{16}}{S_{11}} \\
\frac{S_{12}}{S_{11}} & \beta_{22} & \beta_{23} & 0 & 0 & \beta_{26} \\
\frac{S_{13}}{S_{11}} & \beta_{23} & \beta_{33} & 0 & 0 & \beta_{36} \\
0 & 0 & 0 & \beta_{44} & \beta_{45} & 0 \\
0 & 0 & 0 & \beta_{45} & \beta_{55} & 0 \\
\frac{s_{16}}{S_{11}} & \beta_{26} & \beta_{36} & 0 & 0 & \beta_{66}
\end{array}\right]^{(k)}\left\{\begin{array}{l}
\varepsilon_{1} \\
\sigma_{2} \\
\sigma_{3} \\
\sigma_{4} \\
\sigma_{5} \\
\sigma_{6}
\end{array}\right\}^{(k)}
$$

where

$$
\beta_{i j}^{(k)}=S_{i j}^{(k)}-S_{1 i}^{(k)} / S_{1 j}^{(k)}
$$

\section{Local Analytical Model}

The principle of minimum complementary energy is used with Lekhnitskii stress functions ${ }^{8}$ to determine the stresses in the local analysis. Neglecting body forces, the complementary energy functional for a laminated body consisting of $N$ subvolumes (layers) with volume $V_{(k)}$, is written in Cartesian coordinates as

$$
\Pi_{c}=\sum_{k=1}^{N}\left[\int_{V_{(k)}} \frac{1}{2} S_{i j}^{(k)} \sigma_{i}^{(k)} \sigma_{j}^{(k)}\right] d V-\int_{S_{u}} \tau_{i} \tilde{u}_{i} d S_{u}
$$

In the above expression, $\sigma_{i}^{(k)}$ denotes the stresses, $\tau_{i}$ denotes the surface tractions, $\tilde{u}_{i}$ denotes the prescribed displacements, and $S_{i j}^{(k)}$ denotes the compliance matrix given in Eq. (2). The superscript $(k)$ attached to the variables indicates that the variable is associated with the $k^{t h}$ layer. The displacements $\tilde{u}_{i}$ are prescribed along the boundary segment $S_{u}$. The stress tensor $\sigma_{i}$ satisfies the equilibrium equations within each layer and the traction continuity conditions on inter-layer boundaries, and it is compatible with the prescribed tractions over the boundary segment $S_{G}$. The functional $\Pi_{c}$ must be stationary with respect to the independent admissible variations in the stresses, and the compatibility equations are determined from the resulting EulerLagrange variational equations. The first variation of the complementary energy functional is given as

$$
\begin{aligned}
& \delta \Pi_{c}=\sum_{k=1}^{N}\left[\int_{V_{(k)}} \frac{1}{2} S_{i j}^{(k)} \sigma_{i}^{(k)} \delta \sigma_{j}^{(k)}\right] d V \\
& -\int_{S_{u}} \tilde{u}_{i} \delta \tau_{i} d S_{u}=0
\end{aligned}
$$

The general expression in Eq. (8) is specialized to the generalized plane deformation problem by recalling that the stresses in all layers are independent of the axial coordinate $x$. As a result, only variations of stresses that are independent of $x$ are considered in the analysis. In addition, the integration in $x$ is carried out for a unit length. Carrying out the integration in $x$ reduces the volume integral in Eq. (8) to an area integral in the $y-z$ plane, and the surface integral to a contour integral in the same plane. Making these simplifications, and employing the notation in Fig. 1, Eq. (8) becomes

$$
\begin{aligned}
\delta \Pi_{c} & =\sum_{k=1}^{N} \int_{A_{(k)}} \frac{1}{2} S_{i j}^{(k)} \sigma_{i}^{(k)} \delta \sigma_{j}^{(k)} d A \\
& -\int_{A_{(k)}}\left[\Delta u \delta \sigma_{1}^{(k)}+\Delta v \delta \sigma_{6}^{(k)}+\Delta w \delta \sigma_{5}^{(k)}\right] d A \\
& -\int_{z_{k}}^{z_{k-1}}\left[\tilde{u} \delta \sigma_{6}^{(k)}+\tilde{v} \delta \sigma_{2}^{(k)}+\tilde{w} \delta \sigma_{4}^{(k)}\right] d z=0
\end{aligned}
$$

where $\Delta u, \Delta v, \Delta w$ are the differences between the displacements at the ends $x=0$ and $x=1$, and $A_{(k)}$ denotes the area of the $k^{\text {th }}$ layer in the $x=0$ plane. The longitudinal stress component, $\sigma_{1}^{(k)}$ is determined from Eq. (4).

\section{Layer Stress Assumptions}

The structural analysis proceeds by approximating the stresses within the layers in the two regions shown in Fig. 2. The stress assumptions for the two regions are independent, but are identical in form. Hence, the subsequent discussion is developed for one region. A generic layer in the analysis has thickness $h^{(k)}$ and the upper and lower surfaces are defined by $z^{(k-1)}$ and $z^{(k)}$, respectively, as shown in Fig. 3. A local non-dimensional coordinate $\eta=\left(z-z^{(k)}\right) /\left(z^{(k-1)}-z^{(k)}\right)$ is defined for the analysis. A stress field in the $k^{t h}$ layer satisfying the governing equilibrium equations may be expressed in terms of a pair of stress functions ${ }^{8} \Phi^{(k)}(y, \eta)$ and $\Psi^{(k)}(y, \eta)$ for the generalized plane deformation assumption used in the present analysis such that

$$
\begin{array}{r}
\sigma_{2}^{(k)}=\frac{\partial^{2} \Phi^{(k)}}{\partial^{2} z} \quad \sigma_{3}^{(k)}=\frac{\partial^{2} \Phi^{(k)}}{\partial^{2} y} \\
\sigma_{4}^{(k)}=-\frac{\partial^{2} \Phi^{(k)}}{\partial y \partial z} \\
\sigma_{6}^{(k)}=\frac{\partial \Psi^{(k)}}{\partial z} \quad \sigma_{5}^{(k)}=-\frac{\partial \Psi^{(k)}}{\partial y}
\end{array}
$$


Approximate forms for the stress functions are obtained by assuming a solution for the stress field within a typical layer using separation of variables ${ }^{9}$; i.e.,

$$
\sigma_{i}^{(k)}(y, \eta)=f_{i}^{(k)}(y) g_{i}^{(k)}(\eta) \quad i=I \rightarrow \sigma
$$

(no sum on $i$ )

where $f_{i}^{(k)}(y)$ are unknown functions of the in-plane variable $y$, and $g_{i}{ }^{(k)}(\eta)$ are assumed explicit polynomial shape functions of the through-the-thickness variable $\eta$. The through-the-thickness polynomial functions presented by Yin $^{7}$ and by Rose and Herakovich ${ }^{10}$ are used in the present analysis. In these formulations, expressions for the through-the-thickness shape functions are developed by assuming that the in-plane stress components $\sigma_{2}^{(k)}$ and $\sigma_{6}^{(k)}$ vary linearly through the thickness of a layer in accordance with classical lamination theory. Thus, from Eq. (10), the through-the-thickness shape functions for $\Phi^{(k)}$ in a layer are defined by cubic polynomials in $\eta$, and the through-the-thickness shape functions for $\psi^{(k)}$ in a layer are defined by quadratic polynomials in $\eta$. The assumed polynomial stress functions for each layer $(k)$ used in the present analysis are expressed as

$$
\begin{aligned}
\Phi^{(k)}(y, \eta) & =\left(1-3 \eta^{2}+2 \eta^{3}\right) F_{k}(y) \\
& +\left(\eta-2 \eta^{2}+\eta^{3}\right) h^{(k)} G_{k}(y) \\
& +\left(3 \eta^{3}-2 \eta^{3}\right) F_{k-1}(y) \\
& +\left(\eta^{3}-\eta^{2}\right) h^{(k)} G_{k-1}(y)
\end{aligned}
$$

$$
\begin{aligned}
\Psi^{(k)}(y, \eta) & =\left(1-\eta^{2}\right) L_{k}(y) \\
& +\left(\eta^{2}\right) L_{k-1}(y)+\left(\eta-\eta^{2}\right) h^{(k)} H_{k}(y)
\end{aligned}
$$

where $F_{k}(y), G_{k}(y), L_{k}(y)$ and $H_{k}(y)$ are unknown functions of the in-plane variable $y$.

In addition to satisfying the equilibrium equations within a layer, the stresses must satisfy the traction continuity conditions between layers, and the surface boundary conditions. Continuity of the interlaminar normal stress and the interlaminar shearing stresses at the $k^{t h}$ interface $z=z(k)$ requires that along the interface

$$
\begin{gathered}
\Phi^{(k+1)}=\Phi^{(k)} \quad \boldsymbol{\Phi}, z^{(k+1)}=\Phi, z^{(k)} \\
\Psi^{(k+1)}=\Psi^{(k)}
\end{gathered}
$$

Free surface conditions are also imposed along the top and bottom surfaces of the laminates. For pressureloaded panels, these surfaces may be exposed to normal pressure tractions. However, the magnitude of the pressure load should be small relative to the stresses that develop within the material, and it is ignored in the analysis. Imposing the free surface conditions at the top and bottom surfaces of a laminate with the bottom surface defined by $z=z^{(N)}$ and the top surface defined by $z=z^{(0)}$ yields

$$
\begin{aligned}
& \boldsymbol{\Phi}^{(1)}\left(z=z^{(0)}\right)=\boldsymbol{\Phi}^{(N)}\left(z=z^{(N)}\right)=0 \\
& \Phi, z^{(1)}\left(z=z^{(0)}\right)=\Phi, z^{(N)}\left(z=z^{(N)}\right)=0 \\
& \Psi^{(1)}\left(z=z^{(0)}\right)=\Psi^{(N)}\left(z=z^{(N)}\right)=0
\end{aligned}
$$

In addition, free surface conditions are imposed along surface AB in Fig. 2 and inter-region continuity conditions are imposed along surface $\mathrm{BC}$. These conditions are outlined in the next section.

\section{Application of the Variational Principle}

The unknown functions $F_{k}(y), G_{k}(y)$, $L_{(k)}(y)$, and $H_{(k)}(y)$ are determined by applying Eq. (9) to a generic laminate with edge boundaries at $y=Y_{1}$ and $y=Y_{2}$ (see Fig. 3). In the minimization of the complementary energy, the second and third terms in Eq. (9) contribute only to the particular solutions of the unknown functions. Therefore, these terms are dropped in the formulation of the complementary solution. Substituting for the stresses in terms of the stress functions in the first term in Eq. (9) and integrating by parts with respect to $y$ yields

$$
\begin{aligned}
& \sum_{k=1}^{N} \int_{0}^{h^{(k)}} \int_{Y_{1}}^{Y_{2}}\left\{\left(\beta_{22} \Phi_{z z}+\beta_{23} \Phi_{, y y}+\beta_{26} \Psi_{z z}\right) \delta \Phi_{z z}\right. \\
& +\left(\beta_{23} \Phi_{, y y z z}+\beta_{33} \Phi_{, y y y y}+\beta_{36} \Psi_{, y y z}\right) \delta \Phi \\
& -\left(\beta_{44} \Phi_{, y y z}+\beta_{45} \Psi_{, y y}\right) \delta \Phi_{z} \\
& -\left(\beta_{45} \Phi_{, y y z}+\beta_{55} \Psi_{, y y}\right) \delta \Psi \\
& \left.+\left(\beta_{26} \Phi_{z z}+\beta_{36} \Phi_{y y}+\beta_{66} \Psi_{z}\right) \delta \Psi_{z z}\right\}(d y d z) \\
& +\sum_{k=1}^{N} \int_{0}^{h^{(k)}}\left\{\left(\beta_{23} \Phi_{, z z}+\beta_{33} \Phi_{, y y}+\beta_{36} \Psi_{, z}\right) \delta \Phi_{, y}\right. \\
& -\left(\beta_{23} \Phi_{, y z z}+\beta_{33} \Phi_{, y y y}+\beta_{36} \Psi_{, y z}\right) \delta \Phi \\
& +\left(\beta_{44} \Phi_{, y z}+\beta_{45} \Psi_{, y}\right) \delta \Phi_{z} \\
& \left.+\left(\beta_{45} \Phi_{, y z}+\beta_{55} \Psi_{, y}\right) \delta \Psi\right\}\left.d z\right|_{Y_{1}} ^{Y_{2}} \\
& =0
\end{aligned}
$$

In Eq. (16) the superscripts ( $k$ ) have been dropped for conciseness and a comma denotes partial differentiation. The double integral in Eq. (16) contains the compatibility equations associated with the variations in the stress tensor, and the remaining terms in the equation are the continuity conditions between the regions. Equation 
(16) can be expressed in terms of the $3 n$ unknown functions $F_{k}(y), G_{k}(y)$ and $L_{k}(y)$ and the $n+1$ unknown functions $H_{k}(y)$ by substituting the stress function assumptions of Eqs. (12) and (13) into Eq. (16) and integrating through the thickness of the laminate. Since explicit piecewise continuous functions have been assumed for the through-the-thickness variations of the stresses, the through-the-thickness integration is performed by summing the individual layer integrals in $z^{(k)}$. Carrying out these manipulations gives

$$
\begin{aligned}
& \int_{Y_{1}}^{Y_{2}}\{X\} T\left([W] \frac{d^{4}}{d y^{4}}+[V] \frac{d^{2}}{d y^{2}}+[U]\right)\{\delta X] d y \\
& \quad+\left.\{Y\} T\left([P] \frac{d^{3}}{d y^{3}}+[Q] \frac{d^{2}}{d y^{2}}+[R] \frac{d}{d y}+[S]\right)\{\delta Y\}\right|_{Y_{1}} ^{Y_{2}} \\
& \quad=0
\end{aligned}
$$

where the vectors $\{X\}$ and $\{Y\}$ have the form

$$
\{X\}=\left\{\begin{array}{c}
Y_{1}=F_{1} \\
\cdot \\
X_{1}=F_{1} \\
\cdot \\
\cdot \\
Y_{n}=F_{n} \\
Y_{n+1}=G_{1} \\
\cdot \\
\cdot=F_{n+1} \\
X_{n+1}=G_{1} \\
\cdot \\
\cdot \\
X_{3 n+N} \\
\cdot \\
X_{3 n}=L_{n} \\
X_{3 n+1}=H_{1} \\
Y_{2 n}=G_{n} \\
Y_{2 n+1}=L_{1} \\
\cdot \\
\cdot \\
Y_{3 n}=L_{n} \\
Y_{3 n+1}=H_{1} \\
\cdot \\
\cdot \\
Y_{3 n+N}=H_{N} \\
Y_{3 n+N+1}=\frac{\partial F_{1}}{\partial y} \\
\cdot \\
\cdot \cdot \\
Y_{4 n+N}=\frac{\partial F_{n}}{\partial y} \\
Y_{5 n+N}=\frac{\partial G_{n}}{\partial y}
\end{array}\right\}
$$

\section{Solution Approach}

The matrices $[U],[V]$, and $[W]$ in Eq. (17) have elements that are real-valued constants and are determined by evaluating the double integral term in Eq. (16). The matrices $[P],[Q],[R]$, and $[S]$ are obtained from the other terms in Eq. (16). The individual components of these matrices have been determined using the symbolic manipulation computer code MACSYMA. ${ }^{11}$ These components are extremely lengthy and are not presented. Satisfying Eq. (17) for the arbitrary variations $\delta F_{k}, \delta G_{k}, \delta L_{k}$ and $\delta H_{k}$ yields a system of linear, ordinary differential equations with respect to $y$ with constant coefficients

$$
\left([W] \frac{d^{4}}{d y^{4}}+[V] \frac{d^{2}}{d y^{2}}+[U]\right)\{X\}=\{0\}
$$

with the boundary conditions

$$
\left.\left([P] \frac{d^{3}}{d y^{3}}+[Q] \frac{d^{2}}{d y^{2}}+[R] \frac{d}{d y}+[S]\right)\{Y\}\right|_{Y_{1}} ^{Y_{2}}=\{0\}
$$

There is one set of Eqs. (19) for region A, shown in Fig. 2 , and another set for region $B$. Thus, solutions are obtained for each region as described below, and the solutions are connected by the inter-region continuity conditions that are obtained from Eq. (20). (The interregion continuity conditions are discussed in more detail subsequently). The solution to the homogeneous set of Eqs. (19) is obtained by letting

$$
\{X\}=\{\varphi\} e^{\lambda \bar{y}}
$$

where, for convenience, the following transformations are introduced for the width coordinate $y$

$$
\begin{aligned}
& \bar{y}=y-b \quad \text { for } \mathrm{y} \geq b \\
& \bar{y}=b-y \quad \text { for } \mathrm{y}<b
\end{aligned}
$$

The vertex at $y=b$ (Fig. 2b) is then defined by $\bar{y}=0$, in both regions, and $\bar{y}$ is always positive. Substitution of Eq. (21) into the homogeneous differential equations leads to the eigenvalue problem

$$
[W] \lambda^{4}+[V] \lambda^{2}+[U]=0
$$

which can be written in the standard form

$$
[D]\{\varphi\}=\frac{1}{\lambda^{2}}\{\varphi\}
$$

where $[D]$ is an unsymmetric matrix given by

$$
[D]=\left[C_{0}\right]^{-1}\left[C_{1}\right]=\left[\begin{array}{cc}
{[U]} & {[0]} \\
{[0]} & {[I]}
\end{array}\right]^{-1}\left[\begin{array}{rr}
{[V]} & {[W]} \\
-[I] & {[0]}
\end{array}\right]
$$

The matrices $\left[C_{0}\right]$ and $\left[C_{1}\right]$ have dimension $6 n+1$ by $6 n+1$, and the matrices $[U]$ and $[V]$ have dimension $4 n+1$ by $4 n+1$. The matrix [W], which is associated with the coefficient matrix [W] of the 
operator $d^{4} / d y^{4}$, has dimension $2 n$ by $4 n+1$ and has nonzero elements only in the top $2 n$ by $2 n$ submatrix (in rows and columns corresponding to the functions $F_{k}$ and $G_{k}$ ). The zero matrices [0] have dimension $2 n$ by $4 n+1$ and the identity matrices [I] have dimension $2 n$ by $2 n$.

The eigenvalue problem has been solved by the highly efficient combination shift $\mathrm{QZ}$ algorithm. ${ }^{12}$ For a general laminate, there is a total of $6 n+1$ eigenvalues $\lambda^{2}$; thus there are $6 n+1$ pairs of eigenvalues $\lambda$, that are either real or complex. One component of the pair has a positive real part and the other component of the pair has a negative real part. For the case of a cross-ply laminate, the elastic compliance coefficients $\beta_{26}, \beta_{36}$ and $\beta_{45}$ and $S_{16}$ are zero in all layers, and the system of equations (Eq. (19)) decouples into two systems of equations for the stress functions $\Phi$ and $\Psi$. The system associated with $\Phi$ determines the solution for the in-plane problem (plane strain), and the system associated with $\Psi$ determines the solution for a twisting load. The solution spaces for the eigenvalue problems associated with these two systems have dimension $8 n$ and $4 n+2$, respectively. Eigenvectors associated with the eigenvalues are determined from the homogeneous form of Eq. (19). The total solution is obtained as a superposition of the eigenvectors multiplied by generalized coordinates (complementary solution) plus a particular solution. A particular solution to Eq. (19) can be obtained by assuming $\{X(y)\}$ is constant. A particular solution was obtained from the far-field classical lamination theory stresses, and is outlined in the Appendix.

As mentioned previously, the stiffened panel problem is solved by dividing the structure into two regions. If independent stress functions are assumed for the two regions, two uncoupled eigenvalue problems are obtained when the principle of minimum complementary energy is applied. There are $n$ interfaces in region A and, for a general laminate, $6 n+1$ pairs of eigenvalues contribute to the solution. There are $n_{s}$ interfaces in region $\mathrm{B}$ and hence $6 n_{s}+1$ pairs of eigenvalues. Expressing the solutions in these regions in terms of the eigenvectors and the particular solutions gives

$$
\begin{aligned}
\left\{X^{a}\right\} & =\sum_{i=1}^{6 n+1} a_{i}\left\{\varphi_{i}\right\} e^{\lambda_{i} \bar{y}}+\left\{X_{p}^{a}\right\} \\
\left\{X^{b}\right\} & =\sum_{j=1}^{6 n_{s}+1} b_{j}\left\{\chi_{j}\right\} e^{\beta_{j} y^{\prime}}+\left\{X_{p}^{b}\right\}
\end{aligned}
$$

where the superscripts $a$ and $b$ denote the regions, and the subscript $p$ denotes the particular solution. In the expansions in Eq. (26), only negative eigenvalues are considered since local coordinates $\bar{y}$ have been established with their origins at the thickness discontinuity vertex, and stresses decay with distance from this point. The local coordinates were chosen in this manner to avoid potential numerical overflow problems associated with the evaluation of positive exponential functions. The constants $a_{i}$ and $b_{i}$ are determined by imposing the free edge conditions along the flange edge $\mathrm{AB}$ and at the vertex $B$, and the inter-region continuity conditions along BC (see Fig. 2). The free-surface conditions require

$$
\left\{Y^{a}(\bar{y}=0)\right\}=0 \quad k=1 \rightarrow n_{f}
$$

where $n_{f}=n-n_{s}$. The continuity conditions between the regions are

$$
\begin{gathered}
\left\{Y^{a}(\bar{y}=0)\right\}=\left\{Y^{b}(\bar{y}=0)\right\} \\
\left([P] \frac{d^{3}}{d y^{3}}+[Q] \frac{d^{2}}{d y^{2}}+[R] \frac{d}{d y}+[S]\right)^{a}\left\{Y^{a}(\bar{y}=0)\right\} \\
=\left([P] \frac{d^{3}}{d y^{3}}+[Q] \frac{d^{2}}{d y^{2}}+[R] \frac{d}{d y}+[S]\right)^{b}\left\{Y^{b}(\bar{y}=0)\right\} \\
k=n_{1+f} \rightarrow n_{s}
\end{gathered}
$$

\section{Results}

The approximate analytical solution presented above has been implemented in a FORTRAN code for general laminates. Input to the local analysis program is simple and concise, and includes loading conditions, a description of the geometry, stacking sequence information, and ply material properties. Plies or layers may have different thicknesses and they may be of different materials. The loading conditions for the local analysis are far-field in-plane stresses that are determined from classical lamination theory based on force and moment resultants obtained from a global analysis. Output from the code includes the eigenvalues, eigenfunctions, and constants in the eigenfunction expansion. Once the eigenvalues, the eigenfunctions and the constants in the eigenfunction expansion are determined, transverse and through-the-thickness stress distributions can be easily obtained at any specified location.

Results are presented for two examples. The accuracy of the present analysis of the first example is assessed by comparing the present analysis results with results from an elasticity solution ${ }^{2,3}$ and results from a finite element analysis. Computer execution times are also presented for this example. Further, a brief discussion of the influence of the modeling detail used in the present analysis on the interlaminar stress predictions is presented. The second example illustrates the breadth of information that can be obtained from the present analysis. Stress contour plots, and transverse and through-thethickness stress distributions near the thickness discontinuity are presented. The interaction between the through-the-thickness and the transverse stress gradients in a region near the thickness discontinuity is also discussed. 


\section{Orthotropic Skin and Flange Plates}

The first example application of the analysis is a stiffened graphite-epoxy plate subjected to pure bending by a moment $M_{y}$ applied about the x-axis. The stiffener flange is a rectangular, 8-ply quasi-isotropic $[ \pm 45 / 0 / 90]_{s}$ laminated plate as indicated in the upper sketch of Fig. 4. The skin is an 8-ply orthotropic $\left[ \pm 45 / 90_{2}\right]$ laminated plate that is also rectangular, but is wider than the flange. Each ply in both the flange and skin plates has a uniform thickness $t^{(k)}=0.005$ in. and moduli in the planes of elastic symmetry given by

$$
\begin{gathered}
E_{11}=20 \mathrm{msi} \quad E_{22}=E_{33}=2.1 \mathrm{msi} \\
G_{12}=G_{13}=G_{23}=0.85 \mathrm{msi} \\
v_{12}=v_{13}=v_{23}=0.21
\end{gathered}
$$

where 1,2 , and 3 refer to fiber, transverse, and thickness directions, respectively. To consistently compare results of the present solution with results of an elasticity solution, 2,3 each of the laminates is replaced by an equivalent homogeneous orthotropic plate with smeared compliances obtained from the respective $[A]$ extensional stiffness matrices (see Table 1). The present analysis, however, is not subjected to this limitation; that is, it is possible to model each layer separately. Furthermore, the layers may be monoclinic, and are not limited to specially orthotropic layers.

Typical results for the interlaminar shear stress $\sigma_{y z}$ and the interlaminar normal stress $\sigma_{z z}$ along the skinstiffener interface obtained from the present analysis, from an elasticity solution and from a finite element analysis of the stiffened plate are shown in Figs. $4 \mathrm{a}$ and $4 \mathrm{~b}$, respectively. The interlaminar shear stress $\sigma_{x z}$ for this problem is zero valued, as a result of the uncoupling of the in-plane problem from the torsion problem for specially orthotropic material systems. The elasticity solution is a tedious solution based on complex-variable respresentations of the stress functions and a boundary collocation method. ${ }^{2}$ The finite element analysis was conducted using the displacement based finite element code CLFE2D. ${ }^{13}$ The code CLFE2D was developed for analyzing two-dimensional generalized plane deforma- tion boundary value problems. Thus, only a cross-section of the stiffened plate was modeled in the finite element analysis. The finite element model of the thickness discontinuity region used in the present study has 4033 nodes distributed over 1.5 inches of length. Results from the analytical model presented herein were obtained using 16 layers to model each of the skin and flange plates, and yields a total of 187 eigenvalues in the thick region (region $\mathrm{A}$ ), and 91 eigenvalues in the thin region (region $B$ ). The stresses shown in Figs. $4 a$ and $4 b$ are normalized by $M_{y} t_{s} / 2 I$ and are plotted as a function of the normalized distance $\bar{y}=y / t_{s}$ from the flange termination vertex, where $M_{y}, t_{s}$ and $I$ denote the applied moment, the skin plate thickness, and the moment of inertia of the skin plate, respectively. The solid lines correspond to the present analysis, the dashed lines correspond to the elasticity analysis, and the symbols correspond to the finite element analysis.

The finite element predictions shown in Fig. 4 are discontinuous at the flange-skin interface, and cannot be obtained exactly at the interface without interpolation. Therefore, to provide the most accurate stress data, finite element predictions are plotted for Gauss point locations in the flange and skin plates, just above and below the interface, respectively. Only one value is given for the interface data computed by the elasticity solution and the present analysis, since these analyses satisfy stress continuity across the interface. As the figures indicate, the interlaminar normal stress predictions of all three methods agree very well. The shear stress predictions of the current method and the elasticity solution also agree very well, but there is some discrepancy in the finite element predictions near the vertex of the thickness discontinuity. The correlation between the finite element results, and the present analysis results and the elasticity solution is expected to improve as the finite element mesh is refined. In addition, the results indicate that a significant boundary layer is present in the region near the thickness discontinuity.

Cohen and Hyer ${ }^{2,3}$ have indicated that it is possible for elastic singularities to exist at the thickness discontinuity vertex. The current analysis does not include a singularity explicitly in the formulation. However, singular

TABLE 1. Smeared Elastic Properties for Flange and Skin Plates

\begin{tabular}{lccccccccc}
\hline \hline \multicolumn{1}{c}{ Laminate } & $\begin{array}{c}\mathbf{E}_{\mathbf{x}} \\
(\mathbf{m s i})\end{array}$ & $\begin{array}{c}\mathbf{E}_{\mathbf{y}} \\
(\mathbf{m s i})\end{array}$ & $\begin{array}{c}\mathbf{E}_{\mathbf{z}} \\
(\mathbf{m s i})\end{array}$ & $\begin{array}{c}\mathbf{G}_{\mathbf{x z}} \\
(\mathbf{m s i})\end{array}$ & $\begin{array}{c}\mathbf{G}_{\mathbf{y z}} \\
(\mathbf{m s i})\end{array}$ & $\begin{array}{c}\mathbf{G}_{\mathbf{x y}} \\
(\mathbf{m s i})\end{array}$ & $\begin{array}{c}v_{\mathbf{x z}} \\
(\mathbf{m s i})\end{array}$ & $\begin{array}{c}v_{\mathbf{y z}} \\
(\mathbf{m s i})\end{array}$ & $\begin{array}{c}v_{\mathbf{x y}} \\
(\mathbf{m s i})\end{array}$ \\
\hline Flange $[ \pm 45 / 0 / 90]_{s}$ & 8.05 & 8.05 & 2.16 & 0.85 & 0.85 & 3.09 & 0.164 & 0.164 & 0.303 \\
Skin $\left[ \pm 45 / 90_{2}\right]_{s}$ & 3.83 & 11.71 & 3.83 & 0.85 & 0.85 & 3.09 & 0.176 & 0.107 & 0.201 \\
\hline \hline
\end{tabular}


behavior is predicted by the present analysis. The singular behavior becomes apparent for the example problem by increasing the number of mathematical subdivisions used to model the flange and skin plates. The interlaminar shear and normal stress predictions along the skinstiffener interface are shown in Figs. 5a and 5 b, respectively, for solutions where 4,8 and 16 equal thickness sublayers are used to model each of the flange and skin plates. The solid lines correspond to the 4-layer model, the dashed lines correspond to the 8-layer model, the dashed-dotted lines correspond to the 16-layer model, and the circular symbols correspond to an elasticity solution. The present analysis results based on the 16-layer model agree more closely with the exact elasticity solution in the very local neighborhood of the flange termination at $\bar{y}=0$ than with the corresponding results based on 8layer and 4-layer models. This observation is particularly true for the interlaminar shear stress $\sigma_{y z}$ results. The interlaminar shear stress is equal to its prescribed value of zero along $\bar{y}=0$ for all of the discretization levels shown in Fig. 5. Very steep gradients are encountered in the transverse interlaminar shear stress distribution as the flange termination vertex is approached. As the number of layers in the analytical model increases, the predicted maximum stress increases, and the location of this maximum stress shifts toward the vertex at $\bar{y}=0$, thereby approaching the elasticity solution. The interlaminar normal stress, in contrast, is not prescribed along $\bar{y}=0$ but is singular at the vertex, and the predicted stress at this point increases monotonically as the number of mathematical layers in the model increases. However, the present analysis results away from the vertex are nearly identical for all of the discretization levels that are shown. A similar observation is made for the interlaminar shear stress. These observations are significant from a design perspective, in that depending on the design and failure criteria used in conjunction with the stress analysis, the results based on the 4-layer model may be as adequate for predicting failure as the results based on the 16-layer model, and the 4-layer model takes much less time to execute. Execution times recorded on a Personal Iris 4D/ 35 Workstation for models based on five through-thethickness discretizations are provided in Table 2. These execution times indicate that doubling the number of layers in the analytical model increases the run time by an order of magnitude.

Another method for improving solution accuracy, while keeping computer execution times as low as possible, is to adjust the relative thicknesses of the sublayers used to model the plates rather than increasing the number of sublayers used. ${ }^{14}$ This approach is basically equivalent to mesh refinement. Thick layers can be used for the regions away from the skin-flange interface, and an increased number of thin layers is used near the interface where the gradients are the most pronounced. Interlaminar shear stresses along the skin-stiffener interface are shown in Fig 6. These results were obtained from a model in which the flange and skin plates are represented by two mathematical sublayers. Stress predictions are presented in Fig. 6 as a function of the ratio $t_{1} / t_{2}$, where $t_{1}$ is the thickness of the mathematical layer used to model a portion of the flange or skin plate adjacent to the skin-stiffener interface, and $t_{2}$ is the thickness of the mathematical layer used to model the remaining thickness of the plate. As the thickness of the layer adjacent to the skin-stiffener interface is reduced, the results for $\sigma_{y z}$ from the present analysis approach the elasticity solution. Similar trends have been found for the corresponding interlaminar normal stress $\sigma_{z z}$ results.

\section{Isotropic Plates Subjected to Uniform Extension}

The second example is the analysis of a stiffened aluminum skin panel subjected to an extensional load $N_{y}$ applied to the skin panel. The configuration of the stiffened skin is the same as for the previous example. Both the flange and the skin are 0.04 inches thick and have a modulus of elasticity $E=10 \times 10^{6} \mathrm{psi}$ and a Poisson's ratio $v=0.30$. There is no material-property mismatch between the flange and skin plates. Therefore,

TABLE 2. Execution Times for Five Through-the-Thickness Discretizations

\begin{tabular}{ccccc}
\hline \hline $\begin{array}{c}\text { Number of } \\
\text { layers }\end{array}$ & $\begin{array}{c}\text { Total number } \\
\text { of eigenvalues }\end{array}$ & $\begin{array}{c}\text { Number of skin } \\
\text { plate } \\
\text { eigenvalues }\end{array}$ & $\begin{array}{c}t^{(k)} \\
\text { (inches) }\end{array}$ & $\begin{array}{c}\text { Time } \\
\text { (cpu seconds) }\end{array}$ \\
\hline 4 & 43 & 19 & 0.010 & 2.21 \\
5 & 55 & 25 & 0.008 & 3.82 \\
8 & 91 & 43 & 0.005 & 11.7 \\
10 & 115 & 55 & 0.004 & 26.0 \\
16 & 187 & 91 & 0.002 & 100 \\
\hline \hline
\end{tabular}


this example shows the effect of the geometric discontinuity at the flange termination on the stress field, and provides a simple case for studying the interactions between the transverse and through-the-thickness gradients in the stress field near the thickness discontinuity.

Distributions of the interlaminar shear stress $\sigma_{y z}$ and the interlaminar normal stress $\sigma_{z z}$ obtained from the present analysis are shown in Fig. 7 . The stress components $\sigma_{x y}$ and $\sigma_{x z}$ are zero for this loading condition and material system. Stresses are normalized by the skin-panel far-field stress $\sigma_{y y}$. There is a stress concentration at the flange termination point, where the stresses have a lobe-shaped distribution near the corner of the flange. Large through-the-thickness gradients and inplane gradients in both $\sigma_{y z}$ and $\sigma_{z z}$ are observed near the flange termination point $(\bar{y}=0)$. Away from the flange termination point, the interlaminar stresses are equal to zero.

The through-the-thickness and in-plane gradients of the stress components, and their interactions, are shown explicitly by plots of the stress distributions along the lines $z / t_{s}=0.0, \quad z / t_{s}=-0.025 \quad$ and $z / t_{s}=-0.098$ shown in Fig. 8 by curves A-A, B-B and $\mathrm{C}-\mathrm{C}$, respectively, and at $y / t_{s}=0.022$ (section D-D), shown in Fig. 9. When the flange termination region is approached in the direction $y$, gradients in $\sigma_{y y}$ develop, as shown in Fig. 8, as load is transferred from the stiffened to the unstiffened plate regions. These transverse gradients in $\sigma_{y y}$ are equilibrated by steep through-thethickness gradients in $\sigma_{y z}$, as shown in Fig. 9. The through-the-thickness $\sigma_{y z}$ distribution is self-equilibrating; that is, it integrates to zero through the thickness (its resultant force is zero valued). A through-the-thickness distribution of the interlaminar normal stress $\sigma_{z z}$ is also shown in Fig. 9. The interlaminar normal stress $\sigma_{z z}$ changes sign abruptly as the flange-skin interface is approached; it changes from compression in the flange to tension in the skin. There is also a local variation in the stress distribution as the thickness discontinuity is approached. This local variation is consistent with the contour plots in Fig. 7 and is caused by the different rates of change of $\sigma_{y z}$ with changes in $y$ at different locations through the thickness of the corner region.

\section{Concluding Remarks}

A geometrically linear approximate analysis method for predicting complete three-dimensional stress fields in laminated composite structures with local thickness discontinuities has been presented. The analysis method uses stresses obtained from a global two-dimensional analysis as traction boundary conditions for a local approximate three-dimensional analysis to determine stresses in regions with local thickness discontinuities. The analysis assumes that a state of generalized plane deformation exists. The approximate local analysis is based on the principle of minimum complementary ener- gy and uses stress functions with assumed through-thethickness functional dependence. Input to the local analysis is simple and concise, and includes loading conditions obtained from the global analysis, a description of the geometry, stacking sequence information, and ply material properties. Interfacial and through-the-thickness stress distributions are easily obtained at any specified location, and are not limited to the stiffener-flange interface or interfaces between plies.

The present analysis method has been applied herein to determine the stresses in the flange termination regions of a stiffened laminated composite plate and a stiffened aluminum plate subjected to simple bending and transverse extension loading conditions, respectively. Stress predictions from the present analysis have been compared with corresponding finite element results and results from an elasticity solution, and shown to agree very well. The correlation is shown to improve as the number of layers used in the analytical model is increased. Furthermore, it has been demonstrated that, although the analysis model does not explicitly include a singularity, a singularity appears in the predictions as increasing stresses at the flange-skin interface with increasing number of layers in the analytical model. In addition, changing the relative thicknesses of the mathematical layers used to model the skin and flange elements of a stiffened plate was shown to provide a modeling discretization approach for improving stress predictions without increasing the number of layers and the computational requirements. The computational efficiency and accuracy of the method make it well suited for use in preliminary design and optimization studies of advanced composite structures with local structural details.

\section{Acknowledgment}

A portion of the work reported on herein was supported by the National Research Council.

\section{References}

1. Starnes, J. H., Jr., Knight, N. F., Jr., and Rouse, M., "Postbuckling Behavior of Selected Flat Stiffened Graphite-Epoxy Panels Loaded in Compression," AlAA Journal, Vol. 23, No. 8, August 1985, pp. 1236-1246.

2. Hyer, M. W., and Cohen, D., "Calculation of Stresses in Stiffened Composite Panels," AIAA Journal, Vol., 26, No. 7, July 1988, pp. 852-857.

3. Cohen, D., and Hyer, M. W., "Influence of Geometric Nonlinearities on Skin-Stiffener Interface Stresses," AIAA Paper No. 88-2217, Proceedings of the 29th Structures, Structural Dynamics, and Materials Conference, Williamsburg, VA, April 18-20, 1988, pp. 75-84. 
4. Kan, Han-Pin, Mahler, M. A., Deo, R. B., "Prediction of Stiffener-Skin Separation in Composite Panels," NASA Conference Publication 3104, Part 2, First NASA Advanced Composites Technology Conference, 1990.

5. Kassapoglou, C. and DiNicola, A. J., "Efficient Stress Solutions at Skin-Stiffener Interfaces of Composite Stiffened Panels," AIAA Journal, Vol. 30, No. 7, July 1992, pp. 1833-1839.

6. Kassapoglou, C., "Calculation of Stresses at SkinStiffener Interfaces of Composite Stiffened Panels Under Shear Loads," International Journal of Solids and Structures, Vol. 30, No. 11, 1993, pp. 14911501.

7. Yin, W. L., "Free-Edge Effects In Laminates Under Extension, Bending and Twisting, Part I: A Stress Function Approach," AIAA-91-0959-CP, 1991, pp. 985-995.

8. Lekhnitskii, S. G., Theory of Elasticity of an Anisotropic Elastic Body, Holden-Day, Inc., San Francisco, 1963.

9. Pagano, N. J., "Stress Fields in Composite Laminates," International Journal of Solids and Structures, Vol. 14, 1978, pp. 385-400.

10. Rose, C. A., and Herakovich, C. T., "An Efficient Approximate Solution for Interlaminar Stresses," Composites Engineering, Vol. 3, No. 3, 1993, pp. 271-285.

11. Anon., "VAX UNIX MACSYMA Reference Manual, Version 11," Symbolic, Inc., 1985.

12. Ward, R. C., "An Extension of the QZ Algorithm for Solving Generalized Matrix Eigenvalue Problems," NASA TN D-7305, July 1973.

13. Buczek, M. B., Gregory, M. A., and Herakovich, C. T., "CLFE2D - A Generalized Plane Strain Finite Element Program for Laminated Composites Subjected to Mechanical and Hygrothermal Loading," VPI-E-83-40, Virginia Polytechnic Institute and State University, Blacksburg, VA, 1983.

14. Rose, C. A., "Approximate Analytical Solution for Interlaminar Stresses in Composite Laminates with Extension and Curvature," Ph. D. Dissertation, Civil Engineering, University of Virginia, May 1992.

\section{Appendix}

\section{Elements of particular solution vectors}

The particular solutions for $F_{k}$ and $G_{k}$ in the flange plate are obtained by solving the system of equations:

$$
\begin{aligned}
& {\left[\begin{array}{cccccc}
6 & 2 h^{(1)} & 0 & 0 & \cdot \\
-6 & -4 h^{(1)} & 0 & 0 & \cdot \\
-6 & 4 h^{(2)} & 6 & 2 h^{(2)} & \cdot \\
6 & -2 h^{(2)} & -6 & -4 h^{(2)} & \cdot \\
& & \cdot & & \\
& & \cdot & & \\
& & \cdot & &
\end{array}\right]\left\{\begin{array}{c}
F_{1} \\
G_{1} \\
F_{2} \\
G_{2} \\
\cdot \\
\cdot \\
G_{n_{f} p}
\end{array}\right\}} \\
& =\left\{\begin{array}{c}
\tilde{\sigma}_{2 t}^{(1)}\left(h^{(1)}\right)^{2} \\
\tilde{\sigma}_{2 b}^{(1)}\left(h^{(1)}\right)^{2} \\
\tilde{\sigma}_{2 t}^{(2)}\left(h^{(2)}\right)^{2} \\
\tilde{\sigma}_{2 b}^{(2)}\left(h^{(2)}\right)^{2} \\
\\
\cdot \\
\tilde{\sigma}_{2 b}^{\left(n_{f}\right)}\left(h^{\left(n_{f}\right)}\right)^{2}
\end{array}\right\}
\end{aligned}
$$

where the subscripts $t$ and $b$ denote the top and bottom of a layer, respectively, the tilde denotes classical lamination theory stresses, and $n_{f}$ is the number of layers in the flange plate. The particular solutions for $L_{k}$ and $H_{k}$ in the flange plate are obtained by solving the system of equations:

$$
\begin{aligned}
& {\left[\begin{array}{cccccc}
-2 & -h^{(1)} & 0 & 0 & \cdot & \cdot \\
0 & 1 & 0 & 0 & \cdot & \cdot \\
2 & 0 & -2 & -h^{(2)} & \cdot & \cdot \\
0 & 0 & 0 & 1 & \cdot & \cdot \\
H_{2} \\
H_{2} \\
\cdot \\
H_{1} \\
\cdot \\
H_{n_{f}}
\end{array}\right\}} \\
& =\left\{\begin{array}{c}
\tilde{\sigma}_{6 t}^{(1)} h^{(1)} \\
\tilde{\sigma}_{6 b}^{(1)} h^{(1)} \\
\tilde{\sigma}_{6 t}^{(2)} h^{(2)} \\
\tilde{\sigma}_{6 b}^{(2)} h^{(2)} \\
\cdot \\
\tilde{\sigma}_{6 b}^{\left(n_{f}\right)} h^{(n f)}
\end{array}\right\}
\end{aligned}
$$

The particular solutions in the skin plate are given in Eqs. (32) and (33). 


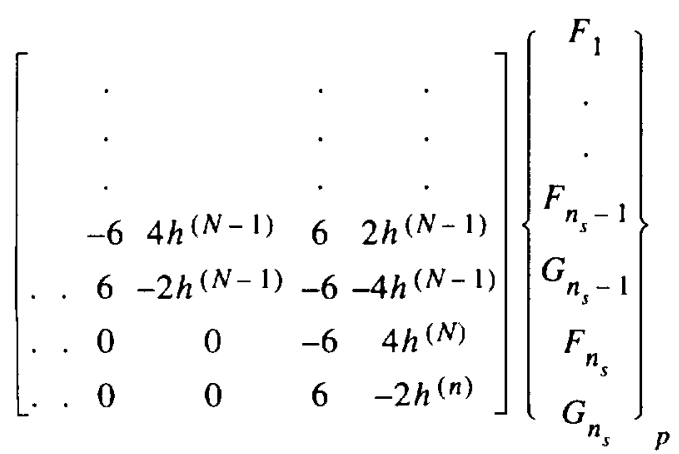

$=\left\{\begin{array}{c}\tilde{\sigma}_{2 t}^{(1)}\left(h^{(1)}\right)^{2} \\ \cdot \\ \tilde{\sigma}_{2 t}^{\left(n_{s}-1\right)}\left(h^{(N-1)}\right)^{2} \\ \tilde{\sigma}_{2 b}^{\left(n_{s-1}\right)}\left(h^{(N-1)}\right)^{2} \\ \tilde{\sigma}_{2 t}^{\left(n_{s}\right)}\left(h^{(N)}\right)^{2} \\ \tilde{\sigma}_{2 b}^{\left(n_{s}\right)}\left(h^{(N)}\right)^{2}\end{array}\right\}$

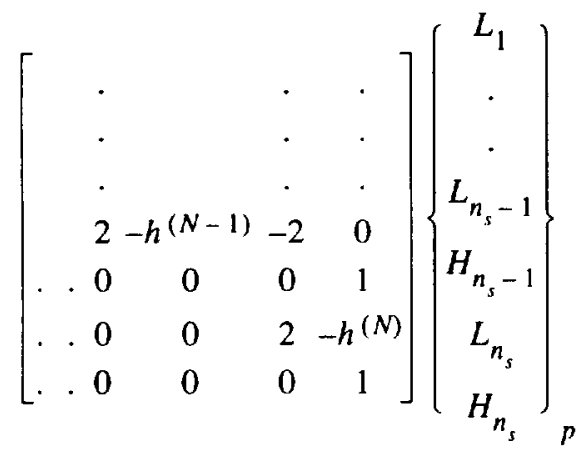

$=\left\{\begin{array}{c}\tilde{\sigma}_{6 t}^{(1)} h^{(1)} \\ \cdot \\ \tilde{\sigma}_{6 t}^{\left(n_{s}-1\right)} h^{(N-1)} \\ \tilde{\sigma}_{6 b}^{\left(n_{s-1}\right)} h^{(N-1)} \\ \tilde{\sigma}_{6 t}^{\left(n_{s}\right)} h^{(N)} \\ \tilde{\sigma}_{6 b}^{\left(n_{s}\right)} h^{(N)}\end{array}\right\}$ 


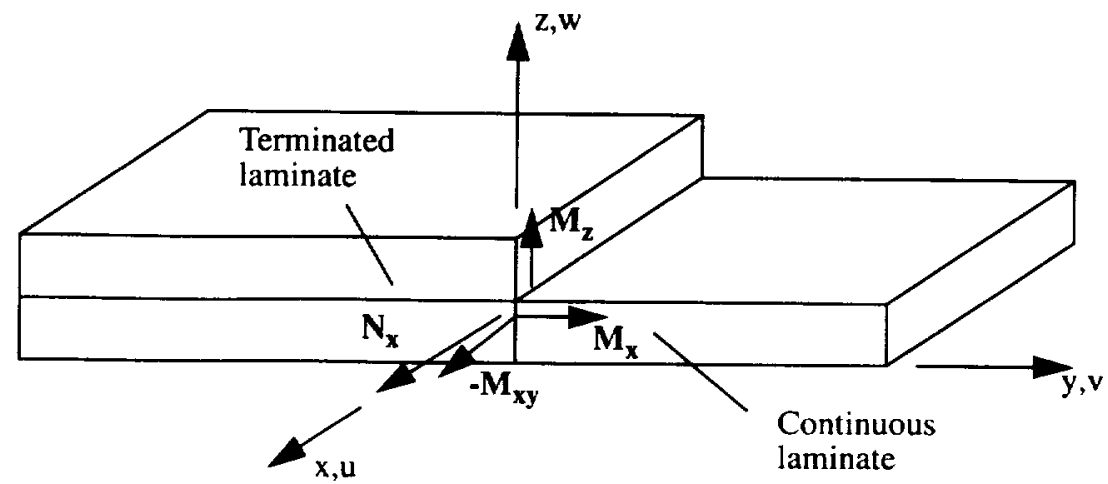

Figure 1. Panel with thickness discontinuity.

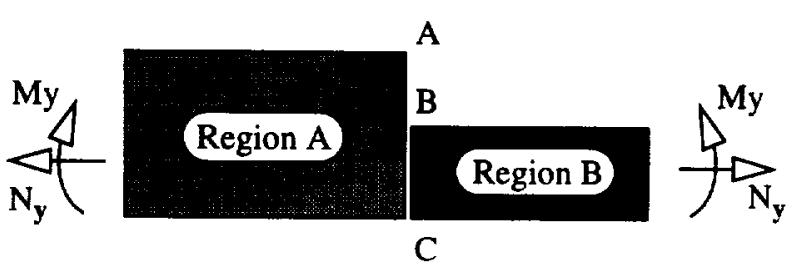

(a) Applied loads

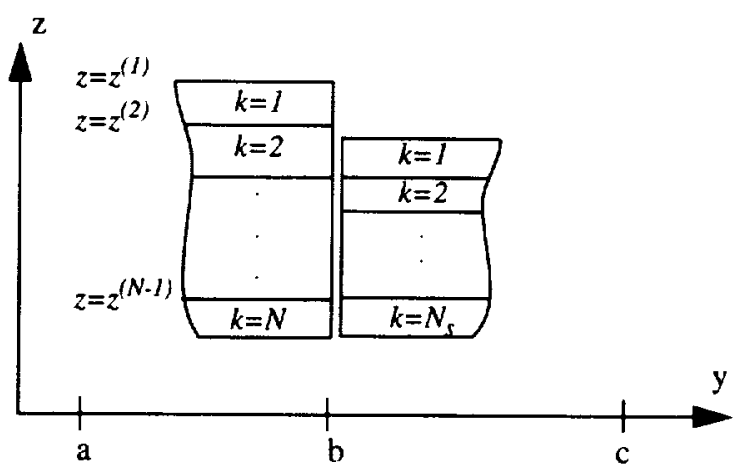

(b) Cross-section model

Figure 2. Cross-section model and applied loads.

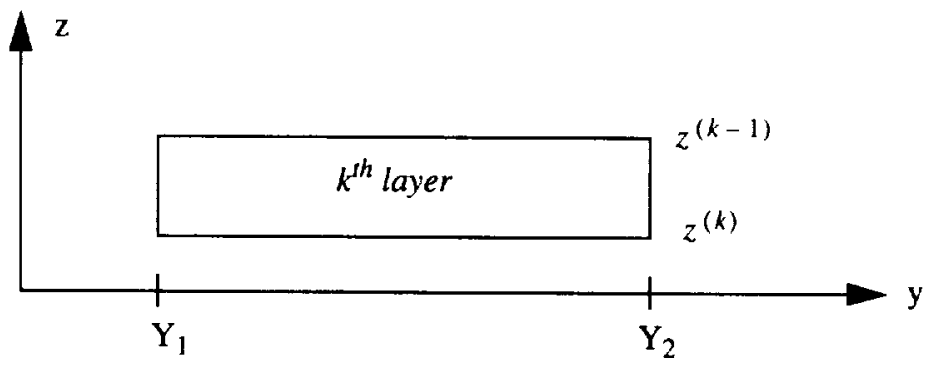

Figure 3. Generic layer. 


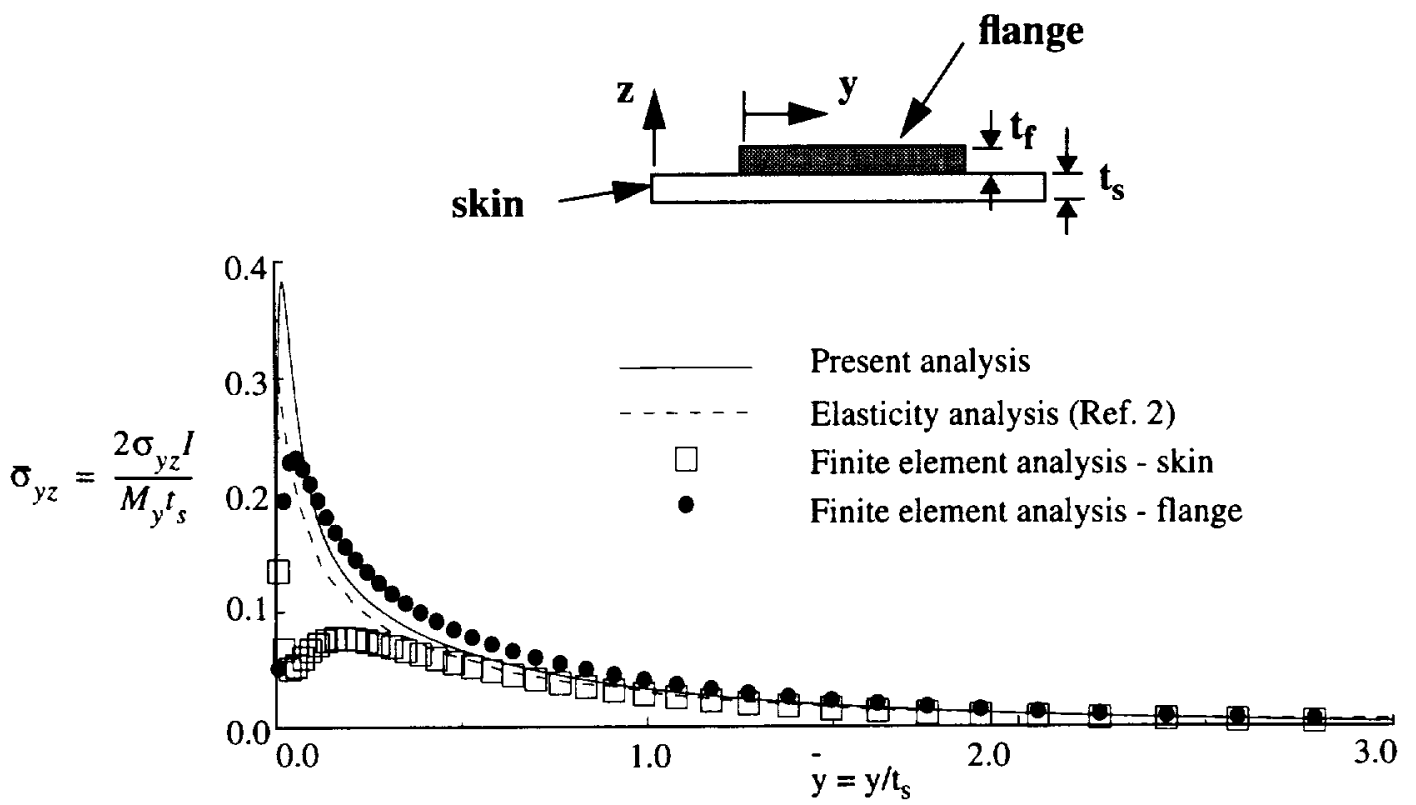

(a) Interlaminar shear stress

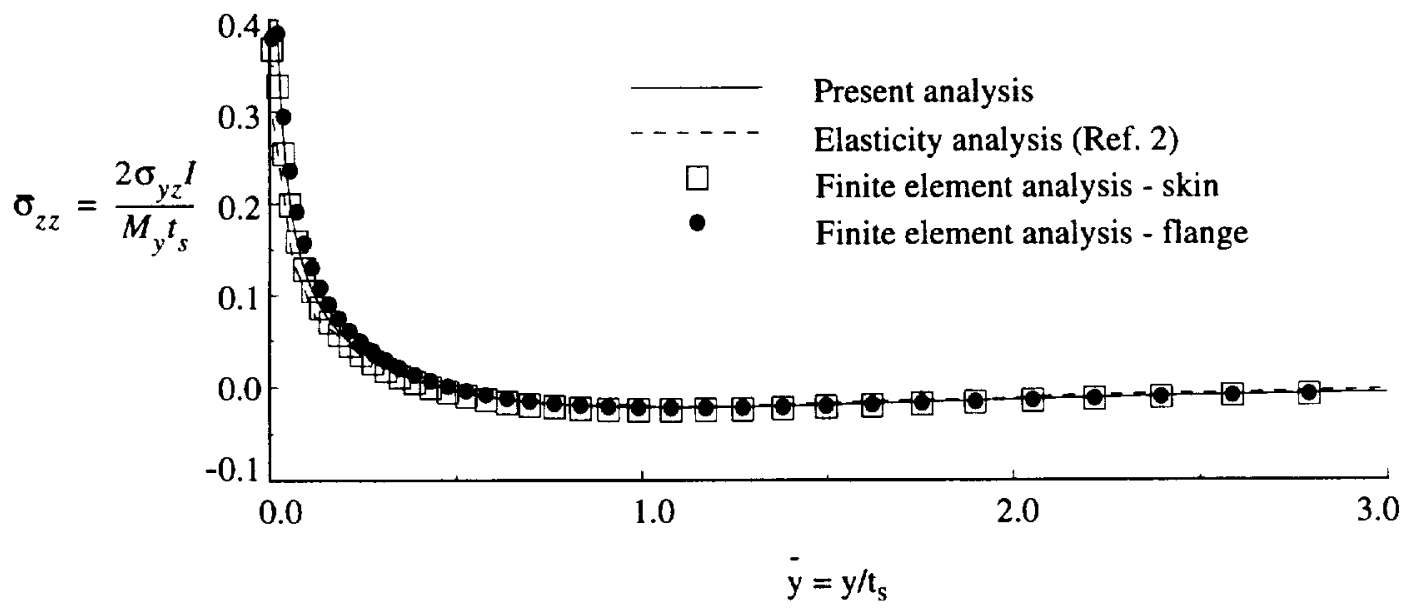

(b) Interlaminar normal stress

Figure 4. Comparison of interlaminar stress distributions along the skin-stiffener interface predicted by the present analysis, an elasticity solution, and a finite element analysis. 


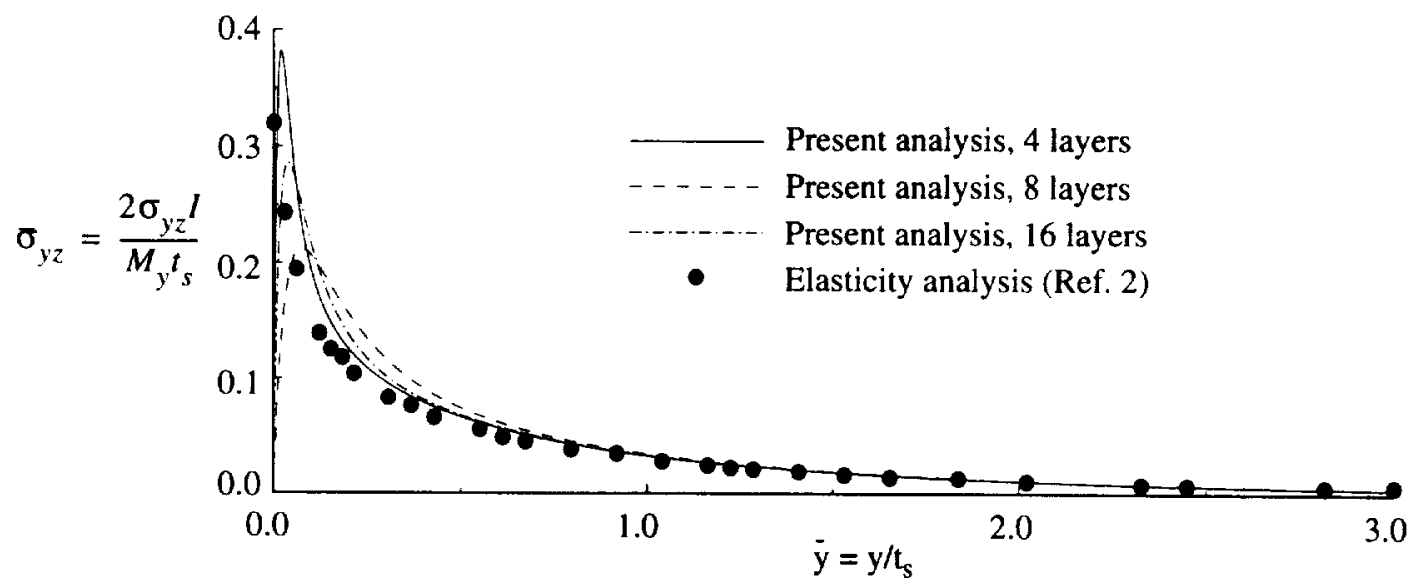

(a) Interlaminar shear stress

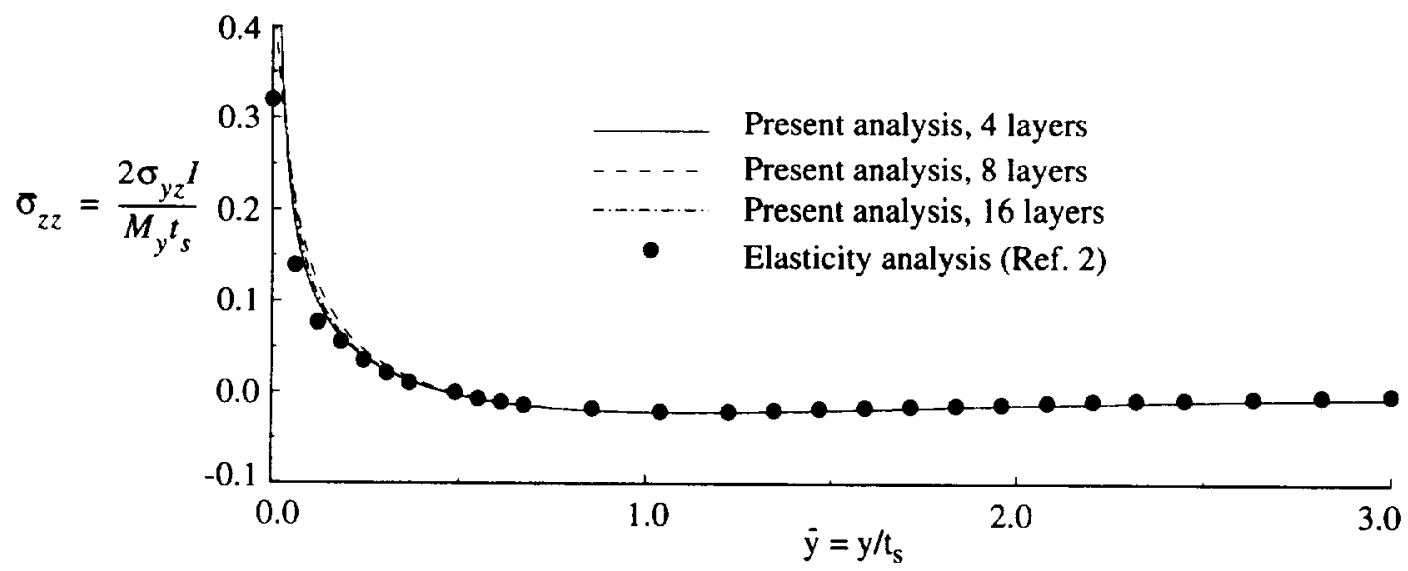

(b) Interlaminar normal stress

Figure 5. Interlaminar stress distributions calculated from an elasticity solution and the present analysis using three through-the-thickness refinements. 


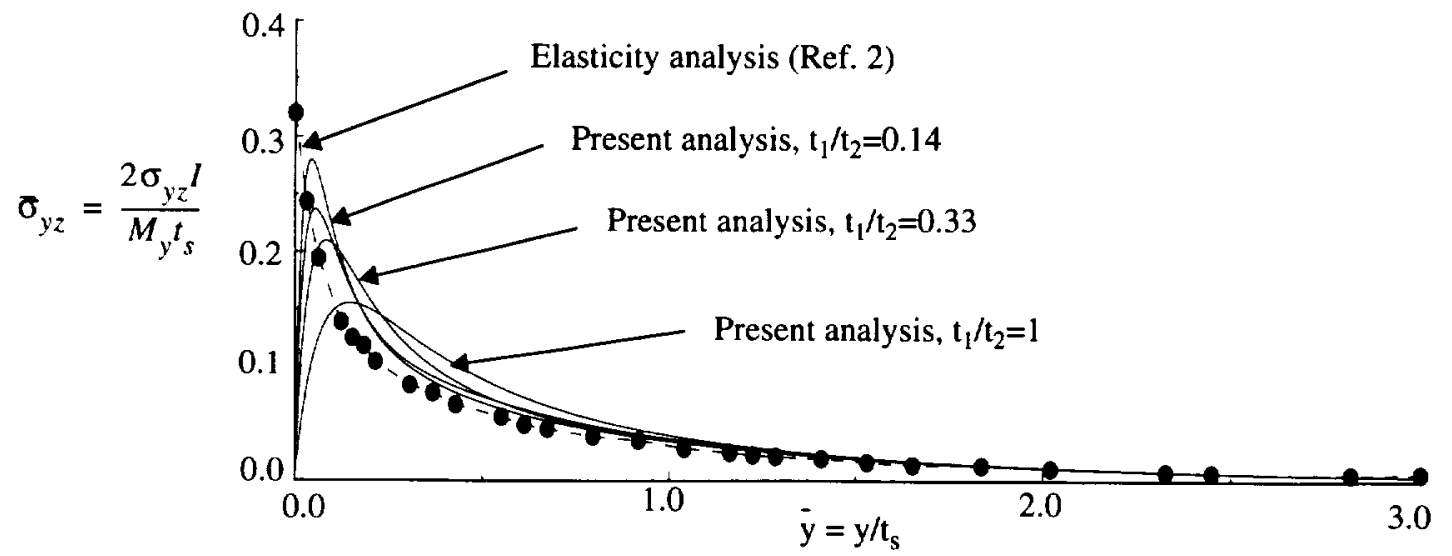

Figure 6. Influence of layer discretization on the interlaminar shear stress predictions along the skin-stiffener interface.

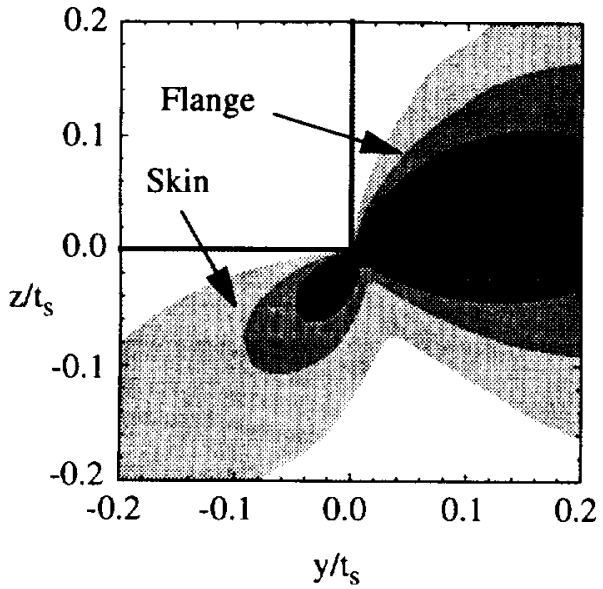

(a) Interlaminar shear stress $\sigma_{y z}$

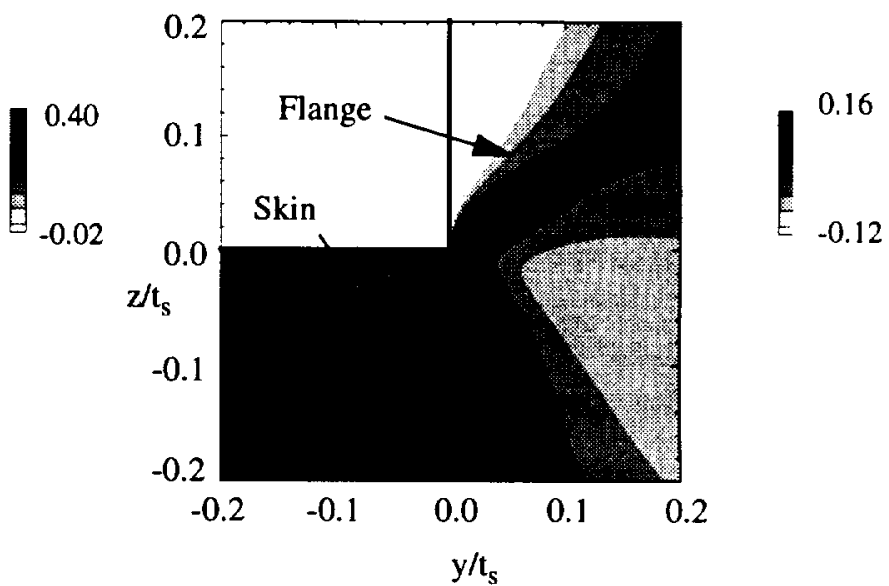

(b) Interlaminar normal stress $\bar{\sigma}_{z z}$

Figure 7. Interlaminar shear and normal stress distributions near a flange termination point of a stiffened aluminum panel subjected to an in-plane skin load $N_{y}$. 


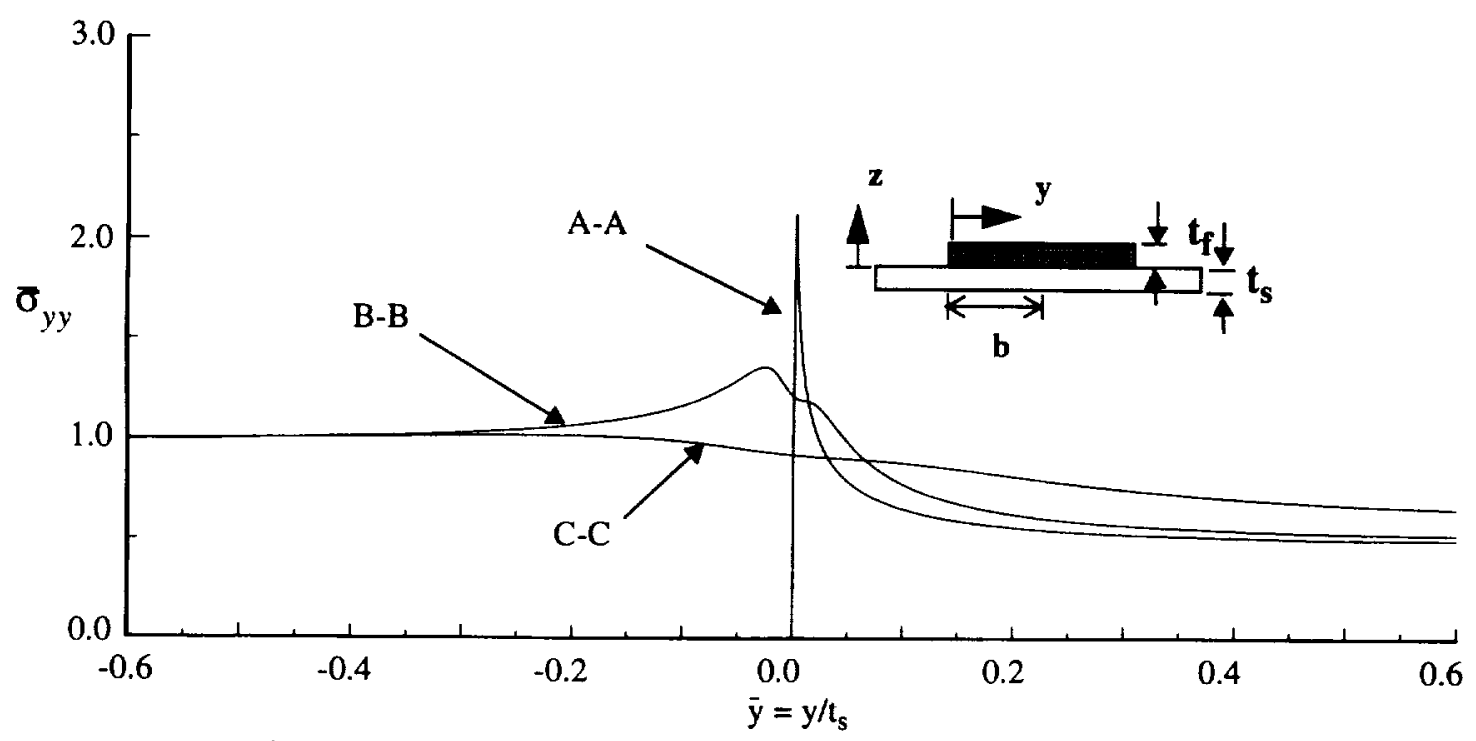

Figure 8. Distribution of transverse in-plane stress at three through-the-thickness locations of a stiffened aluminum panel subjected to an in-plane skin load $\mathrm{N}_{\mathbf{y}}$.
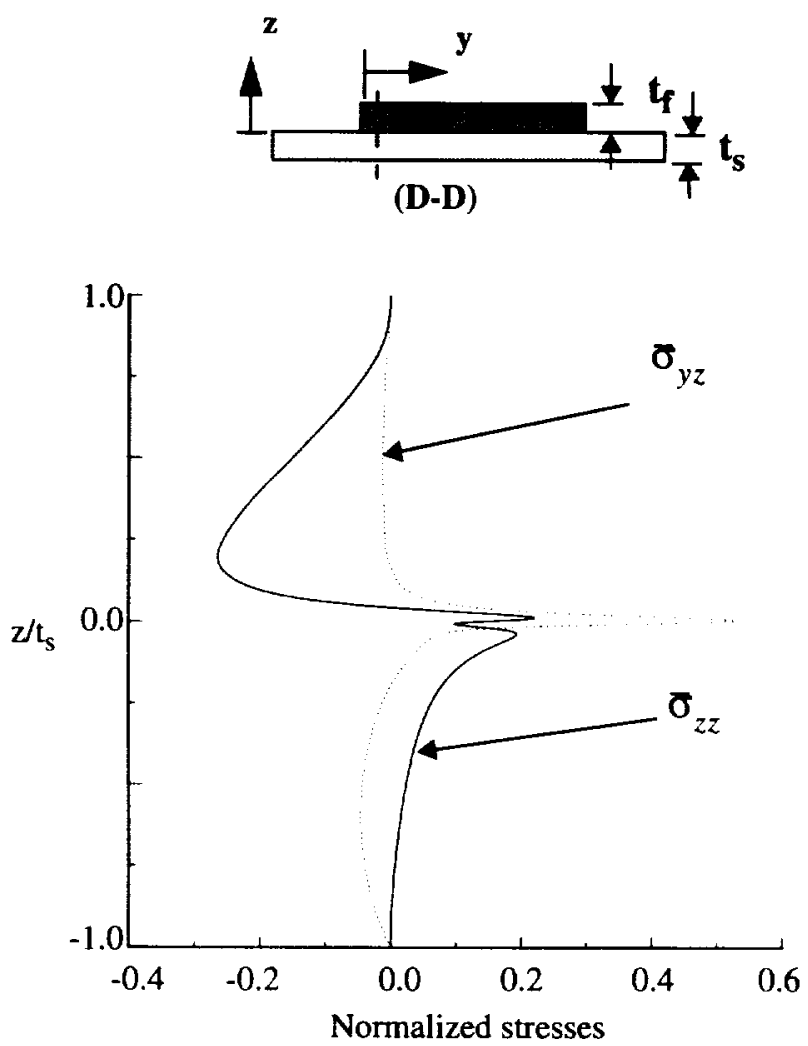

Figure 9. Through-the-thickness interlaminar shear and normal stress distributions at section (D-D) of a stiffened aluminum panel subjected to an in-plane skin load $\mathrm{N}_{\mathrm{y}}$ 